\title{
Insulin Acting as a Modulator of Feeding through the Hypothalamus
}

\author{
Y. Oomura and H. Kita \\ Department of Physiology, Faculty of Medicine, Kyushu University, Fukuoka, Japan
}

Summary. To understand the functional role of insulin in the control of feeding, biochemical and physiological studies were performed in the rat.

1) Insulin content in the brain was much higher than that in the blood, and was extremely variable from animal to animal.

2) Specific binding sites of insulin in the brain were most abundant in the hypothalamus and olfactory bulb.

3) Neither insulin content nor binding sites in the brain was significantly affected by peripheral insulin concentration.

4) Activity of glucoreceptor neurons in the ventromedial hypothalamus (VMH) was facilitated by simultaneous application of insulin and glucose, but inhibited by insulin alone.

5) Activity of the glucose-sensitive neurons in the lateral hypothalamus (LHA) was facilitated by insulin in a dose-dependent manner.

6) Stimulation of the ventral part of the LHA accelerated pancreatic vagal nerve activity. Stimulation of the dorsal part of the LHA and the VMH was inhibitory.

7) Pancreatic splanchnic nerve activity during LHA stimulation tended to show inhibition, but sometimes was modulated by the stimulus frequency. Both inhibition and facilitation were observed in the activity in response to VMH stimulation.

Key words: Brain insulin, brain insulin binding sites, ventromedial hypothalamus, lateral hypothalamus glucoreceptor neuron, glucose-sensitive neuron, pancreatic vagal nerve, pancreatic splanchnic nerve, insulin secretion

The significance of insulin in energy metabolism and control of food intake have been extensively studied. Insulin may act not only on tissues such as liver and fat tissue but also on the central nervous system. For example, administration of insulin into the carotid artery or into the cerebrospinal fluid caused a decrease in the level of peripheral blood glucose, possibly via vagal nerve stimulation of insulin secretion $[1,2]$. The response of the olfactory bulb and amygdala to olfactory stimulation was altered by insulin injection [3]. Activity of the hypothalamus was affected by systemic administration of insulin through the carotid artery [4]. All of these insulin effects are closely linked to control of feeding behaviour. To clarify the neuronal mechanisms underlying these functions, we have investigated: 1) the existence of insulin and insulin binding sites in the hypothalamus, and their properties; 2) the characteristic actions of insulin on chemo-sensitive neurons in the hypothalamus; 3) the hypothalamic control of insulin secretion via pancreatic autonomic nerve activity. All data point to the importance of insulin in the control of feeding.

\section{Insulin Content and Binding Sites in Rat Brain}

Until recently, insulin activity in the central nervous system was not considered to be worthy of note. Now, however, we have many reasons to study the insulin actions in the brain. The existence of insulin and insulin binding sites in the brain have been reported $[5,6,7]$.

\section{High Insulin Content in the Brain}

Surprisingly, the insulin content of the brain is much higher than that of the blood, and it is not affected by changes in peripheral blood insulin concentration. These facts, shown by Havrankova et al. [6], seem important to us, so we conducted similar experiments, and generally confirmed their results.

Table 1 shows the insulin content of extracts of whole brain, and plasma insulin concentrations in control (fed), fasting, and streptozotocin-treated rats. 
Table 1. Insulin content in rat brain, and blood. Fasting group were food-deprived for $48 \mathrm{~h}$. Streptozotocin-treated group: Streptozotocin was injected $(60 \mathrm{mg} / \mathrm{kg}$, i. p.) five days prior to experiment. Controls and streptozotocin-treated group were allowed free access to standard rat chow. Whole brain was extracted with acid ethanol and insulin content determined by radio-immunoassay as described elsewhere [7]

\begin{tabular}{lcc}
\hline $\begin{array}{l}\text { Rat } \\
\text { number }\end{array}$ & $\begin{array}{l}\text { Brain insulin content } \\
\text { (mU/kg wet weight) }\end{array}$ & $\begin{array}{l}\text { Plasma insulin level } \\
(\mathrm{mU} / \mathrm{l})\end{array}$ \\
\hline Control group & & \\
1. & 185.6 & 49.4 \\
2. & 85.2 & 38.2 \\
3. & 81.2 & 27.2 \\
4. & 65.6 & 24.7 \\
5. & 104.4 & 37.0 \\
6. & 160.0 & 36.2 \\
Fasting group & & \\
7. & 59.4 & 4.6 \\
8. & 654.0 & 12.5 \\
9. & 63.4 & 9.5 \\
10. & 53.2 & 5.2 \\
11. & 69.6 & 7.5 \\
12. & 175.4 & 5.0 \\
Streptozotocin-treated group & \\
13. & 99.0 & 9.5 \\
14. & 86.2 & 17.0 \\
15. & 65.6 & 7.8 \\
16. & 68.8 & 5.3 \\
17. & 63.0 & 2.8 \\
18. & 88.8 & 6.2 \\
19. & 40.0 & 10.0 \\
20. & 1587.0 & 10.8 \\
\hline
\end{tabular}

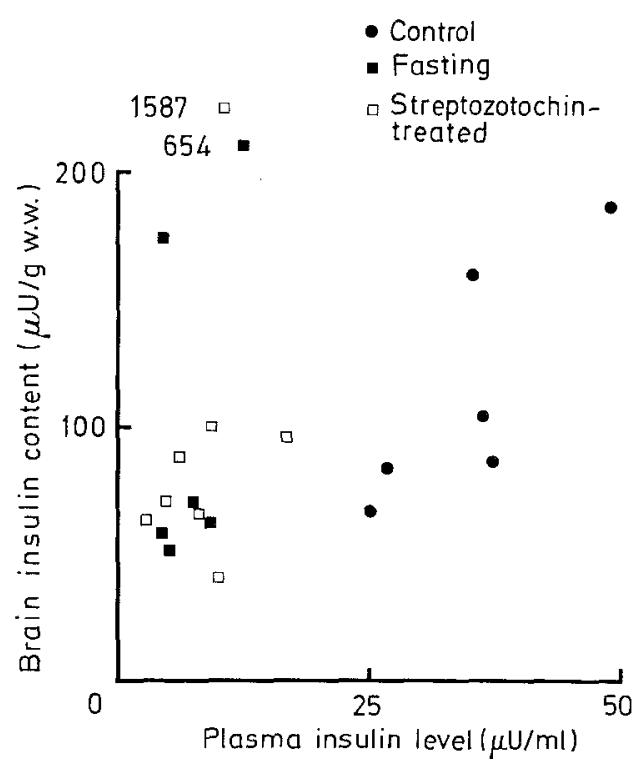

Fig. 1. Correlation between plasma insulin concentration and brain insulin content in rats. Plots made from Table 1 [7]
Brain insulin appears indistinguishable from plasma insulin in molecular size, immunoreactivity with guinea pig antiserum, and biological activity [7]. Plasma insulin concentration of all the fasting and streptozotocin-treated rats was much lower than that of the control animals. However, insulin content of the brain did not decrease in parallel with blood insulin levels. Insulin content per $\mathrm{kg}$ wet weight brain tissue was always higher than plasma insulin concentration per litre. This evidence of the stability of brain insulin concentration agrees with the results of Havrankova et al. [6]. However brain insulin content in the present experiments was much lower than that reported by Havrankova et al.

It is puzzling that the amount of brain insulin was found to be quite variable from animal to animal (Table 1). For example, rat number 8 in the fasting group and rat number 20 in the streptozotocintreated group had very much higher insulin content in the brain, but the plasma insulin concentration of these rats was comparable to that of the other rats in the fasting and streptozotocin treated groups. From the finding of the unaltered brain insulin level after the experimental conditions, the intracerebral biosynthesis of insulin seems to be a possibility. There is, however, another possibility. The brain may accumulate insulin from circulating blood by high affinity uptake and storage sites. Figure 1 shows the correlation between plasma and brain insulin levels. If we exclude the unusual animals which have very high levels of brain insulin, then this graph does suggest some dependence of brain insulin content on plasma insulin level. Van Houten has shown clear evidence of insulin uptake from blood into the brain [8]. More detailed analysis might be necessary to elucidate the origin of brain insulin. We are currently investigating this problem in more detail.

\section{Distribution of Insulin Receptors in the Brain and the Effects of Fasting and Streptozotocin-Treatment}

Havrankova et al. [9] found insulin binding sites in many regions of the rat brain, most abundantly in the olfactory bulb. Furthermore, they demonstrated that the binding sites are independent of peripheral blood insulin concentration [5]. Our studies confirm their results, and further show that the hypothalamus is densely populated with insulin binding sites. The effects of fasting and steptozotocin treatment on insulin binding sites have also been studied.

Figure 2 shows the specific binding of ${ }^{125} \mathrm{I}$-insulin to plasma membrane in various portions of rat brain, and the effects of fasting and streptozotocin treatment. ACTH, growth hormone, tri-iodothyronine, 


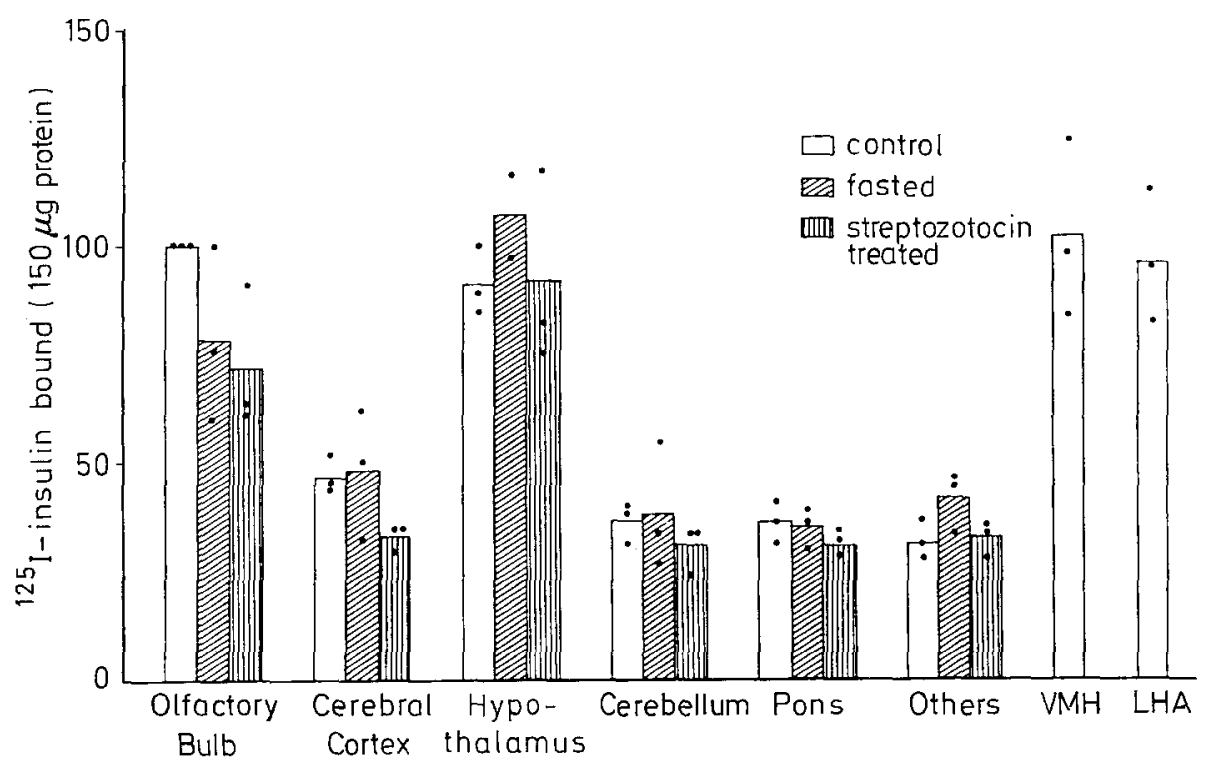

Fig. 2. Distribution of insulin binding to plasma membrane of various portions of the brain from control, fasting, and streptozotocin-treated animals. In some instances the ventromedial (VMH) and lateral hypothalamus (LHA) were isolated from the hypothalamus. Preparation of the plasma membrane fraction and the assay for specific insulin binding sites were performed as described elsewhere [7]. ${ }^{125}$ I-insulin binding was expressed as percentage of specific binding (100\%) in olfactory bulb in the control animals [7] glucagon, and vasopressin did not displace ${ }^{125} \mathrm{I}$-insulin bound to plasma membrane [7]. From this evidence, the binding sites for ${ }^{125} \mathrm{I}$-insulin were considered to be specific to blood-borne insulin. The concentration of binding sites for insulin was highest in the olfactory bulb and the hypothalamus. Concentrations in the ventromedial hypothalamus (VMH), and the lateral hypothalamus (LHA) were virtually the same as that of the entire hypothalamus. The lowest levels were found in the cerebellum and the pons. Fasting and streptozotocin treatment did not produce any observable change in insulin binding sites in the brain.

A new discovery in our study was that both VMH and LHA contain abundant insulin binding sites. It is interesting that the areas containing the most insulin binding sites, such as the VMH, LHA and olfactory bulb, are related to food intake.

\section{Direct Action of Insulin in the Hypothalamus}

The physiological significance of insulin in the brain, especially in the hypothalamus, has been suggested by many studies $[1,4,10,11,12]$. We have summarised below the evidence for the direct action of insulin on hypothalamic neurons.

We have studied the chemo-sensitivity of neurons by means of electrophoretic application from multibarrel micropipettes [14]. These pipette-electrodes permit direct observation of the effects of chemicals on single neurons. In the hypothalamus, two different kinds of chemo-sensitive neurons respond to insulin. One is a glucoreceptor neuron in the VMH, and the other a glucose-sensitive neuron in the LHA.

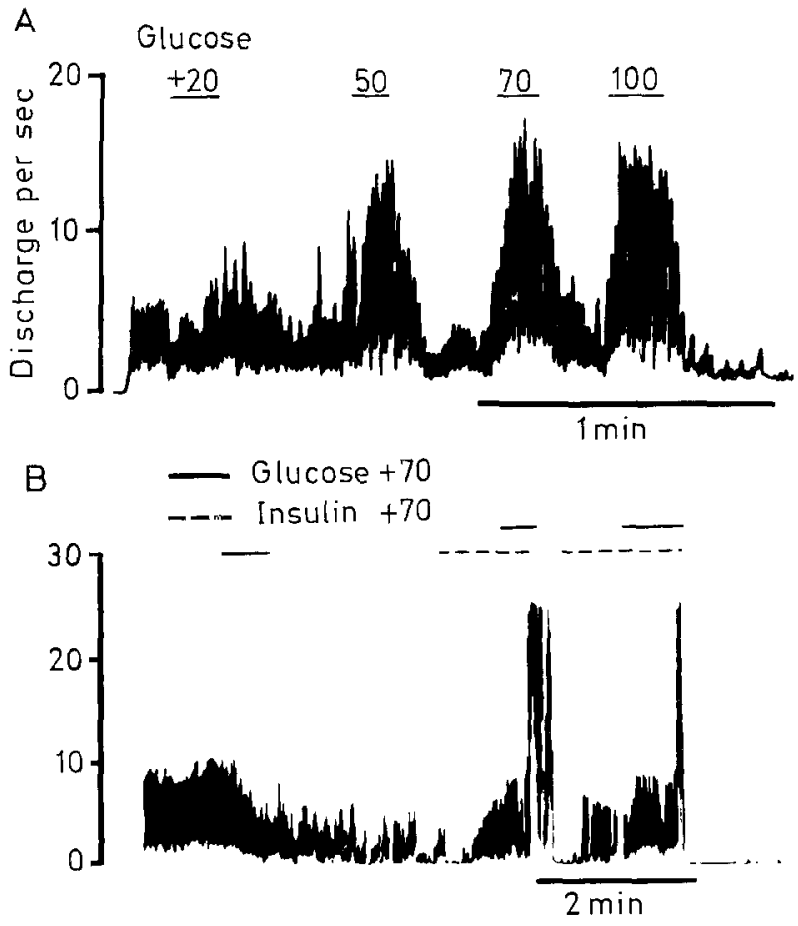

Fig. $3 \mathrm{~A}$ and B. Ratemeter plots of discharges in typical rat VMH glucoreceptor neurons. A Firing frequency was increased by glucose applications in dose-responsive manner. Amount of glucose application is expressed in $\mathrm{nA}$, and duration indicated by horizontal lines. Note the relatively long latency of a few records which indicate glucose, rather than a current effect. B Insulin application decreased firing frequency, and glucose plus insulin strongly facilitated the activity [13]

\section{Insulin Effects on Glucoreceptor Neuron}

The response of glucoreceptor neurons to the electro-osmotic application of glucose is an increase in firing frequency (Fig. 3A). In this type of neuron, 
A Glutamate -20

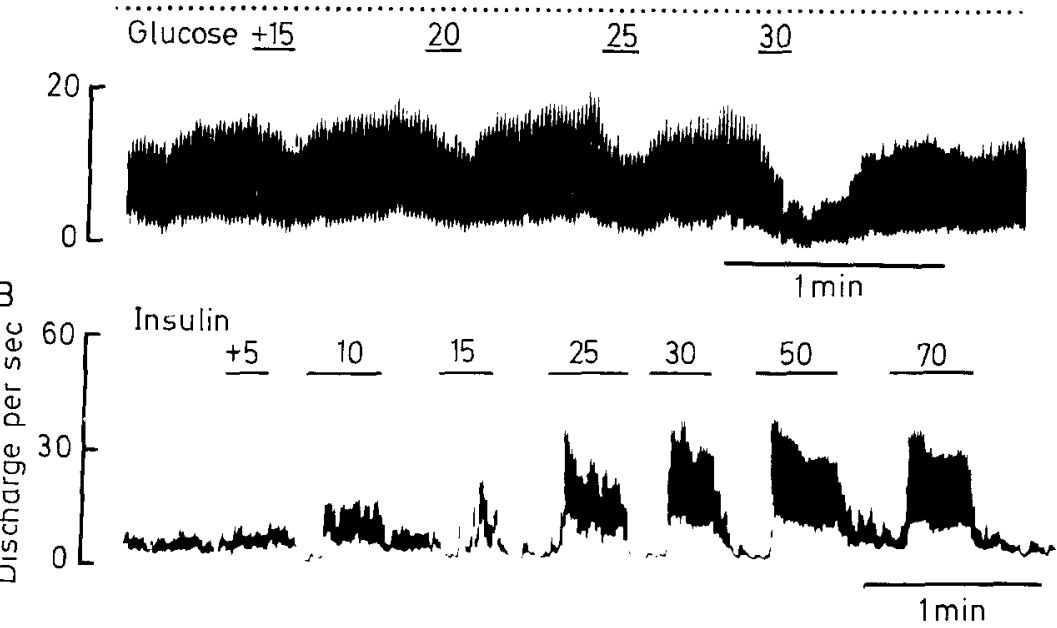

Fig. $4 \mathrm{~A}$ and $\mathrm{B}$. Ratemeter plots of activity of a glucose-sensitive neuron in the LHA. A Glucose application decreases firing rate in dose-responsive manner. B Insulin effect on a glucose-sensitive neuron is facilitation of activity in a dose-responsive manner [13]

electro-osmotic application of insulin enhances the effects of glucose when both glucose and insulin are applied simultaneously, although insulin application alone tends to inhibit the neuron activity (Fig. 3 B) [13].

There are few available reports of electrophysiological studies which explain the above observations. Studies of muscle membrane in rats show that insulin induces membrane hyperpolarization with acceleration of energy dependent $\mathrm{Na}^{+}-\mathrm{K}^{+}$pump activity [16]. This kind of hyperpolarization might explain the inhibitory effect of insulin application alone to VMH glucoreceptor neurons. Insulin is also reported to enhance the binding of the glucose analogue, goldthioglucose, in the VMH of mice $[10,11]$. At the specific glucoreceptive sites of the VMH neuron membrane, insulin might increase binding, or uptake of excess glucose, thus causing the increase in firing frequency seen in this type of neuron.

\section{Insulin Effects on Glucose-Sensitive Neuron}

Glucose-sensitive neurons in the LHA have characteristics which are completely different from those of the $\mathrm{VMH}$ glucoreceptor neurons. Figure $4 \mathrm{~A}$ is a typical example of an LHA glucose-sensitive neuron. Direct application of glucose decreases the firing frequency in a dose-responsive manner. In the LHA, only glucose-sensitive neurons were affected by the electro-osmotic application of insulin as illustrated in Figure 4B [13].

The dose-response relation of the insulin effect indicates that one insulin molecule can react with one membrane receptor site [21]. The threshold level for the insulin effect was approximately $5 \times 10^{-9}$ IU
[13]. This evidence implies the existence of specific insulin receptor sites on LHA glucose-sensitive neurons.

Aside from these clear-cut insulin effects on chemo-sensitive neurons in the hypothalamus, the effects of insulin on glucose metabolism in the central nervous system are still controversial.

In studies on slices of rat spinal cord and cerebellum, insulin caused increased glucose uptake, and the importance of normal membrane organization for this insulin action was emphasized $[17,18]$. In other brain regions, no effect of insulin on glucose incorporation has been reported $[19,20]$. The presence of insulin and its specific receptor is a prerequisite for the action of insulin, but may not necessarily imply that the tissue is regulated by this hormone. For example, the erythrocytes and the kidney have insulin receptors but no clear insulin dependent metabolic process has been found. The insulin effects on the hypothalamus that are presented in this paper appear not to be directly coupled with neuronal glucose metabolism, but stem from special action on the neuron surface membrane. Insulin may have an entirely different functional role in the brain from that found peripherally. It might act as a neuromodulator or a neurotransmitter.

\section{Insulin as a Regulater of Food Intake and Energy Metabolism}

It has been questioned whether insulin could reach the brain tissue through the blood-brain barrier. Studies with the use of ${ }^{125}$ I-insulin have suggested that insulin can penetrate into the brain to some extent [20]. In some areas of the hypothalamus, the 
nerve cell membranes are directly attached to capillary walls without an intervening astroglial membrane suggesting the absence of the blood brain barrier in these areas [13]. Therefore, it cannot be denied that the activity of the hypothalamus could be influenced by blood-borne insulin.

Glucoreceptor neurons in the VMH and glucosesensitive neurons in the LHA monitor the blood levels of glucose as well as insulin, and change their activity. Activity of these neurons is also affected by non-esterified fatty acids and other blood-borne metabolites [21]. It is now widely accepted that the feeding behavior of mammals is governed by the LHA (acting as a feeding center) and the VMH (as a satiety center) with their reciprocal activity changes [22]. Before feeding, the activity of the LHA is increased and that of the $\mathrm{VMH}$ is decreased. After feeding these relations reverse. Furthermore, stimulation of the LHA can produce initiation of feeding [23] and lesion of the $\mathrm{VMH}$ produces hyperphagia and obesity in animals [24]. Stimulation of the VMH and lesion of the LHA can produce opposite effects from the above $[25,26]$. It is quite likely that these chemo-sensitive neurons play physiologically significant roles in the regulation of food intake and energy metabolism by responding to levels of bloodborne chemicals.

\section{Hypothalamic Control of Insulin Secretion from the Pancreas}

Secretion of insulin from the pancreas is considered to be primarily controlled by blood-borne chemicals such as glucose and adrenaline, and modulated by autonomic neuronal activity. Many previous studies have suggested the importance of the hypothalamus in the modulation of pancreatic hormone secretion via the autonomic nervous system, and in the regulation of metabolism. Lesions of the VMH enhanced the responsiveness of the pancreatic islets of Langerhans to insulin secretory stimulation [27, 28, 29]. Also, hyperphagia and obesity following VMH lesions were prevented by vagotomy $[30,31,32]$ or removal of the pancreas from its usual neural influence [33]. By contrast, VMH stimulation decreased plasma insulin level [34]. The LHA is also involved in pancreatic hormone secretion. Effects similar to those of VMH lesions can be produced by LHA stimulation $[29,34,35]$. Both sympathetic and parasympathetic systems appear to be involved in the LHA lesion syndrome $[36,37]$.

The effects of peripheral autonomic nerve stimulation on insulin secretion have been thoroughly studied. Insulin secretion was decreased by splan- chnic nerve stimulation [38, 39] and increased by vagal stimulation [39, 40, 41, 42]. However, the neuronal mechanisms underlying these hypothalamic and/or peripheral nerve effects on pancreatic hormone secretion were still obscure. To clarify these mechanisms, the influence of descending pancreatic autonomic nerve activity after bilateral hypothalamic stimulation has been studied using the urethanechloralose anaesthetized rat. Recording was as reported previously [43].

\section{Effects of VMH Stimulation on Pancreatic Nerve Activity}

The activity of the pancreatic branch of the vagal nerve was clearly descreased by VMH stimulation. Figure 5 (top) is an example of the response: spike discharge rate of the vagal nerve decreased as a function of stimulus frequency and/or intensity. This inhibitory effect was produced only by stimulation of the $\mathrm{VMH}$, and not by stimulation of other medial hypothalamic regions [43].

The inhibition of pancreatic vagal activity by VMH stimulation may be a reason for the decrease in insulin secretion during VMH stimulation which has

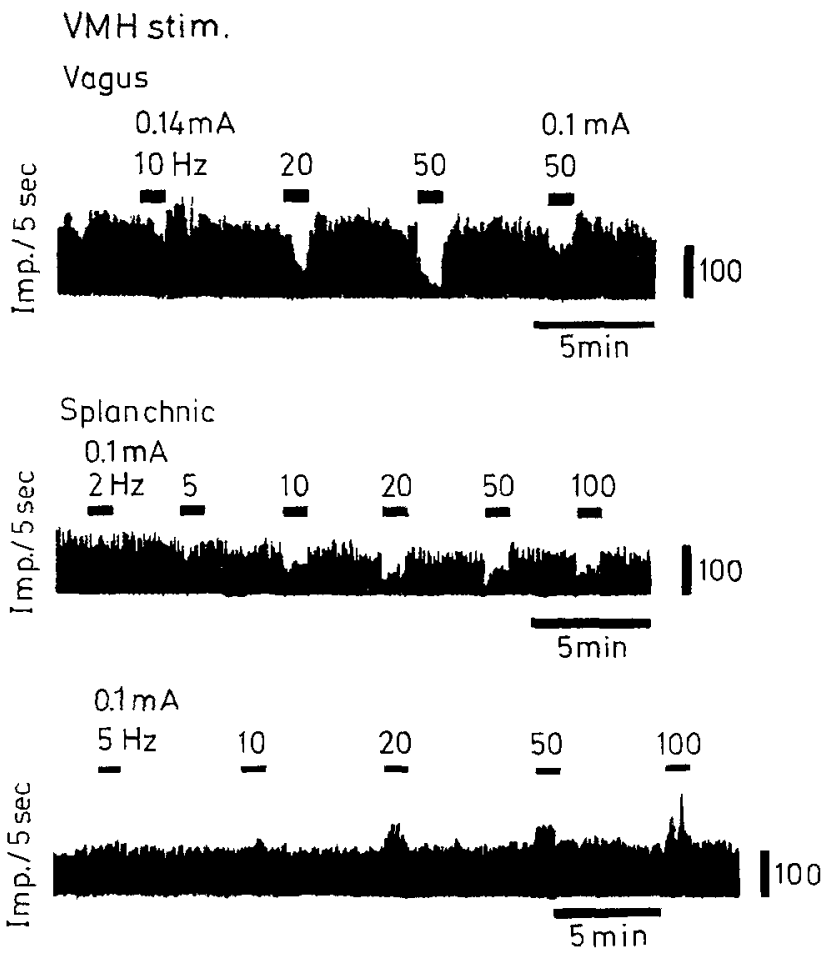

Fig. 5. Example of pancreatic nerve response to bilateral VMH stimulation. Top: Vagal nerve firing decreased by repetitive stimulation. Splanchnic nerve activity inhibited (middle) in half of observed cases. Other half of cases were facilitated (bottom) [43] 
LHA stim.

Vagus
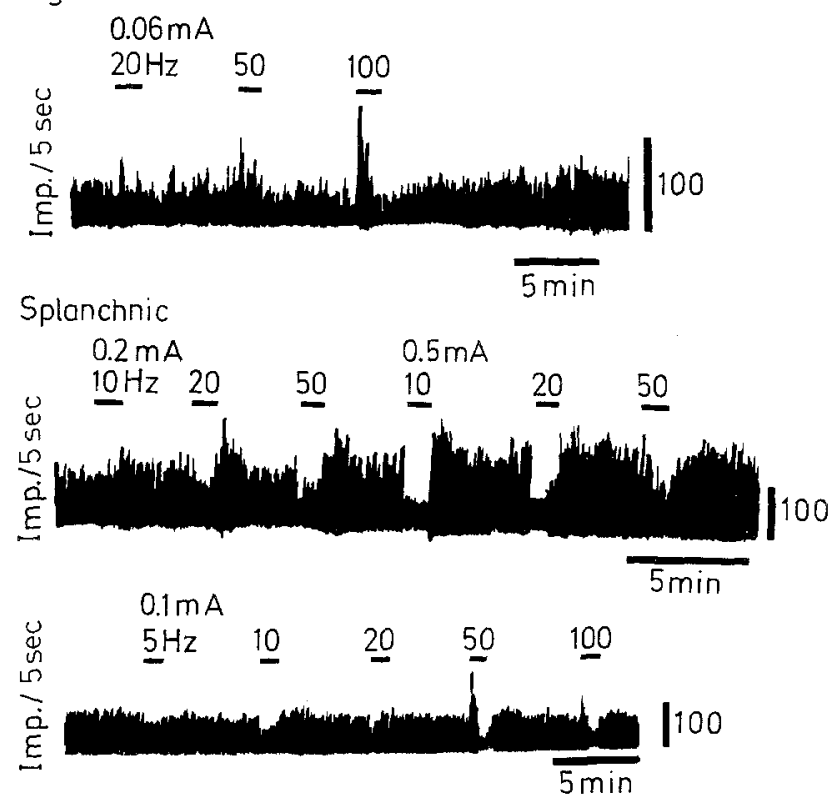

Fig. 6. Example of pancreatic nerve response to LHA stimulation. Top: Vagal nerve response. Activity increased at all stimulating frequencies. Middle and bottom: Splanchnic nerve response. Low frequency stimulation inhibited activity but response was not simple. Response magnitude decreased by high current stimulation (middle). Sometimes, high frequency stimulation produced brief facilitation preceding inhibition (bottom) [43]

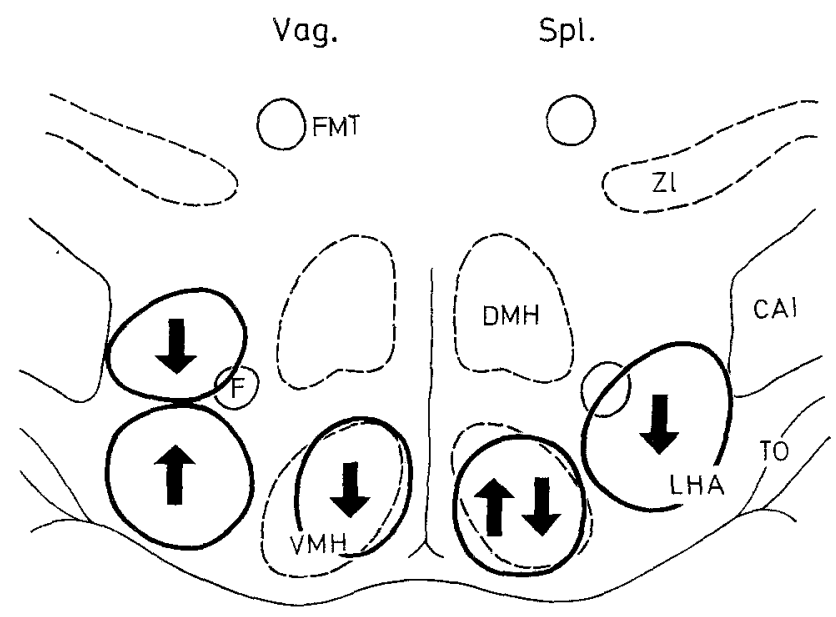

Fig. 7. Illustration of the sites in the hypothalamus which affected pancreatic vagal (left) and splanchnic (right) activity. Vagal nerve was facilitated by ventral LHA stimulation, but inhibited by VMH and dorsal LHA stimulation. Both facilitation and inhibition were observed in splanchnic nerve, in response to VMH stimulation; LHA stimulation tended to inhibit this activity. FMT, fasciculus mamillothalamicus; ZI, zona incerta; CAI, capsula interna; DMH, dorsomedial hypothalamus; F, fornix; TO, optic tract; VMH, ventromedial nucleus; LHA, lateral hypothalamus [43] been reported [34]. In contrast, lesions of the VMH may result in hyperactivity of this nerve due to disinhibition. This could be one of reasons why vagotomy prevents the obesity which is induced by VMH lesions.

Activity of the pancreatic branch of the splanchnic nerve was also affected only by VMH stimulation, and the effect differed from case to case. In half the cases, nerve activity was decreased by VMH stimulation, but in the other half it was increased by the stimulation, as shown in Figure 5 (middle and bottom). In both cases, the response was graded with stimulus frequency.

The above results indicated that pancreatic autonomic nerve activity is modulated by the VMH, while the dorsomedial hypothalamic region does not affect pancreatic nerve activity.

\section{Effects of LHA Stimulation on Pancreatic Nerve Activity}

The response of the pancreatic branch of the vagal nerve to LHA stimulation depended on the stimulating sites. When stimulus electrodes on both sides of the brain were placed in the ventral part of the LHA, this nerve activity was facilitated, as illustrated by Figure 6 (top). Stimulation of the dorsal part of the LHA tended to decrease the firing frequency of the pancreatic vagal nerve. Differentiation of the areas in the LHA has been suggested by other studies [44].

The glucose-sensitive neurons which were mentioned previously in this paper exist mainly in the ventral part of the LHA, and other chemo-sensitive neurons, such as osmo-sensitive neurons, exist in the dorsal part of the LHA $[13,21]$. Furthermore, the ventral LHA receives mainly inhibitory influences from the prefrontal cortex in the rat, while the dorsal LHA receives facilitatory fibres $[44,45]$. These observations may suggest that there are functional difference between those two areas.

The activity of the pancreatic branch of the splanchnic nerve in response to LHA stimulation tended to show inhibition. But the response was not simple, as illustrated by Figure 6 (middle and bottom). The usual inhibitory responses to low frequency stimulation sometimes changed in response pattern to high frequency stimulation. In some cases brief facilitation was preceded by inhibition, and even if the pattern did not alter the response magnitude was often decreased.

It has been reported that vagotomy does not eliminate insulin secretion. This result could be brought about by disinhibition of the pancreas through the splanchnic nerve since LHA stimulation 
inhibits splanchnic activity and increased splanchnic activity inhibits pancreatic insulin secretion.

The regional organization of the hypothalamus, based on its effects on pancreatic autonomic nerve activity, is summarized in Figure 7 . The region that produced excitation of the pancreatic vagal nerve was largely limited to the ventral part of the LHA, and the region that produced inhibition included both the VMH and the dorsal part of the LHA.

The high activity in the VMH and the low activity in the LHA, which are caused by high blood glucose and insulin after feeding, may inhibit pancreatic vagal nerve activity. This inhibition could play an important role in the negative feed-back control of insulin secretion. The existence of a neural feed-back system is suggested by the high insulin secretion which occurs after feeding in the VMH lesioned rat [29]. Before feeding, the activity of the LHA is increased and that of the VMH decreased by the high nonesterified fatty acid levels in the blood [21]. The pancreatic vagal nerve activity may thus be facilitated at this time and enhance the responsiveness of the pancreatic islets of Langerhans to insulin secretory stimulation. This possibility is quite likely since $\mathrm{VMH}$ lesions as well as LHA stimulation produced hyperresponsiveness of pancreatic insulin secretion to a chemical stimulus such as glucose $[12,14,43]$.

Our present studies showed clear relations between hypothalamic and vagal nerve activity, but the relation between pancreatic sympathetic nerve and hypothalamic activity was complicated, since the effects were not simply correlated with stimulus sites in the hypothalamus, and changed with stimulus frequency. More extensive study is needed to clarify these points. But it can at least be said that whether using stimulation or lesioning to relate hypothalamic and pancreatic functions, the treatment must be carefully limited to small, specific sites.

Acknowledgement. We thank Profs. A. Simpson, A. Niijima and Y. Sakamoto, and Drs. S. Aou, K. Yamabe and H. Yoshimatsu for help in preparation of this manuscript. The work was partly supported by grant in aid for Scientic Research 244021 and 448103 and grant in aid for Special Project Research 212110 and 313408 from the Ministry of Education, Science and Culture.

\section{References}

1. Szabo O, Szabo AJ (1975) Studies on the nature and mode of action of the insulin-sensitive glucoregulator receptor in the central nervous system. Diabetes 24: $328-336$

2. Woods SC, Porte DJr (1975) Effect of intracisternal insulin on plasma glucose and insulin in the dog. Diabetes 24: 905-909

3. Cain DP (1975) Effects of insulin injection on responses of olfactory bulb and amygdala single units to odors. Brain Res 99: 69-83

4. Anand BK, Dua S, Singh B (1961) Electrical activity of the hypothalamic 'feeding centers' under the effect of changes in blood chemistry. Electroencephalogr. Clin Neurophysiol 13: 54-59

5. Havrankova J, Schmechel D, Roth J, Brownstein M (1978) Identification of insulin in rat brain. Proc Natl Acad Sci USA 75: 5737-5741

6. Havrankova J, Roth J, Brownstein M (1979) Concentrations of insulin and of insulin receptors in the brain are independent of peripheral insulin levels. J Clin Invest 64: 636-642

7. Sakamoto Y, Oomura Y, Kita H, Shibata S, Suzuki S, Kuzuya $\mathrm{T}$, Yoshida $\mathrm{S}$ (in press) Insulin content and insulin receptors in the rat brain. Effect of fasting and streptozotocin treatment. Biomed J 1

8. Van Houten M, Posner BI, Koprina BM, Brawer JR (1979) Insulin-binding sites in the rat brain: In vivo localization to the circumventricular organs by quantitative radio-autography. Endocrinology 105: 666-673

9. Havrankova T, Roth J, Brownstein M (1978) Insulin receptors are widely distributed in the central nervous system of the rat. Nature 272: 827-829

10. Debons AF, Krimsky I, From A (1970) A direct action of insulin on the hypothalamic satiety center. Am J Physiol 219: 938-943

11. Debons AF, Krimsky I, From A, Roger JC (1969) Rapid effects of insulin on the hypothalamic satiety center, Am J Physiol 217: 1114-1118

12. Storlien LH, Bellingham WP, Martin GM (1975) Localization of CNS glucoregulatory insulin receptors within the ventromedial hypothalamus. Brain Res 96: 156-160

13. Oomura Y (1973) Central mechanism of feeding. In: Kotani M (ed) Advances in biophysics, vol. 5. Tokyo University Press, Tokyo, p 65-142

14. Oomura Y, Ono T, Ooyama H, Wayner MJ (1969) Glucose and osmosensitive neurons of the rat hypothalamus. Nature 222: 282-284

15. Creese R, Jenden DJ (1968) Sodium fluxes in diaphragm muscle and the effects of insulin and serum proteins. J Physiol (Lond) 197: 255-278

16. Zierler K (1966) Possible mechanisms of insulin action on membrane potential and ion fluxes. Am J Med 40: 735-739

17. Rafaelsen OJ (1958) Action of insulin on isolated rat spinal cord. Lancet II: 941-943

18. Rafaelsen OJ (1961) Action of insulin on glucose uptake of rat brain slices and isolated rat cerebellum. $J$ Neurochem 7: 45-51

19. Quastel, JH (1969) Carbohydrate metabolism in the nervous system. In: Bourne GH (ed) The structure and function of nervous tissue, vol 3. Academic Press, New York, p 61-107

20. Goodner CJ, Berrie MA (1977) The failure of rat hypothalamic tissues to take up labeled insulin in vivo and to respond to insulin in vitro. Endocrinology 101: 605-612

21. Oomura Y, (1976) Significance of glucose, insulin and free fatty acid on the hypothalamic feeding and satiety neurons. In: Novin D, Wyrwicka W, Bray G (eds) Hunger: Basic mechanism and clinical implications. Raven Press, New York, p $145-157$

22. Oomura $\mathrm{Y}$, Ooyama $\mathrm{H}$, Naka F, Yamamoto T, Ono T, Kobayashi N (1969) Some stochastical patterns of single unit discharges in the cat hypothalamus under chronic conditions. Ann NY Acad Sci 157: 666-689

23. Larsson S (1954) On the hypothalamic organization of the nervous mechanism regulating food intake. Acta Physiol Scand 32 [Suppl 115]: 1-63

24. Hetherington AW, Ranson SW (1940) Hypothalamic lesions and adiposity in the rat. Anat Rec 78: 149-172

25. Anand BK, Brobeck JR (1951) Hypothalamic control of food intake in rats and cats. Yale J Biol Med 24: 123-140

26. Mayer J, Marshall NB (1956) Specificity of gold thioglucose 
for ventromedial hypothalamic lesions and hyperphagia. Nature 178: 1399-1400

27. Louis-Sylvestre J (1978) Relationship between two stages of prandial insulin release in rats. Am J Physiol 235: E 103-E 111

28. Martin JM, Konijnendijk W, Bouman PR (1974) Insulin and growth hormone secretion in rats with ventromedial hypothalamic lesions maintained on restricted food intake. Diabetes 23: 203-208

29. Steffens AB, Mogenson GJ, Stevenson JAF (1972) Blood glucose, insulin, and free fatty acids after stimulation and lesions of the hypothalamus. Am J Physiol 222: 1446-1452

30. Eng R, Gold RM, Sawchenko PE (1978) Hypothalamic hypo activity prevented but not reversed by subdiaphragmatic vagotomy. Physiol Behav 20: 637-641

31. Inoue S, Bray GA (1977) The effects of subdiaphragmatic vagotomy in rats with ventromedial hypothalamic lesions and obesity. Endocrinology 100: 108-114

32. King BM, Carpenter RG, Stamoutsos BA, Frohman LA, Grossman SP (1978) Hyperphagia and obesity following ventromedial hypothalamic lesions in rats with subdiaphragmatic vagotomy. Physiol Behav 20: 643-651

33. Inoue S, Bray GA, Mullen YS (1977) Effect of transplantation of pancreas on development of hypothalamic obesity. Nature 266: 742-744

34. Frohman LA, Bernardis LL (1971) Effect of hypothalamic stimulation on plasma glucose, insulin, and glucagon levels. Am J Physiol 221: 1596-1603

35. Ball GG (1974) Vagotomy: Effect on electrically elicited eating and self-stimulation in the lateral hypothalamus. Science 184: 484-485

36. Opsahl CA (1977) Sympathetic nervous system involvement in the lateral hypothalamic lesion syndrome. Am J Physiol 232: R 128-R 136

37. Opsahl CA, Powley TL (1977) Body weight and gastric acid secretion in rats with subdiaphragmatic vagotomy and lateral hypothalamic lesions. J Comp Physiol Psychol 91: 1284-1296
38. Girardier L, Seydoux J, Campfield LA (1976) Control of A and $B$ cells in vivo by sympathetic nervous input and selective hyper or hypoglycemia in dog pancreas. J Physiol (Paris) 72: 801-814

39. Miller RE (1975) Neural inhibition of insulin secretion from the isolated canine pancreas. Am J Physiol 229: 144-149

40. Bergman RN, Miller RE (1973) Direct enhancement of insulin secretion by vagal stimulation of the isolated pancreas. Am J Physiol 225: 481-486

41. Kaneto A, Miki E, Kosaka K (1974) Effects of vagal stimulation on glucagon and insulin secretion. Endocrinology 95: $1005-1011$

42. Ujnäs-Wallensten K, Nilsson G (1978) A quantitative study of the insulin release induced by vagal stimulation in anesthetized cats. Acta Physiol Scand 102: 137-142

43. Kita H, Niijima A, Oomura Y, Ishizuka S, Aou S, Yamabe K, Yoshimatsu H (1980) Pancreatic nerve response induced by hypothalamic stimulation in rats. Brain Res Bull 5 [Suppl 4]: 163-168

44. Oomura Y, Takigawa M (1976) Input-output organization between the frontal cortex and the lateral hypothalamus. In: Desiraju $\mathrm{T}$ (ed) Mechanisms in transmission of signals for conscious behaviour. Elsevier, Amsterdam, p 163-192

45. Kita $\mathrm{H}$, Oomura $\mathrm{Y}$, Ishizuka $\mathrm{S}$ (in press) Functional synaptic interconnections between lateral hypothalamus, and frontal and gustatory cortices in rat. In: Katsuki Y, Sato M, Norgren R (eds) Brain mechanisms of sensation. John Wiley \& Sons, New York

Y. Oomura

Department of Physiology

Faculty of Medicine

Kyushu University 60

Fukuoka 812

Japan

\section{Discussion after Oomura's Presentation}

Van Houten: How do you think glucose actually alters the electrical activity of individual neurons in the VMH?

Oomura: We have found that neurons in the VMH have specific glucoreceptors on their membranes. There are unique proteins on these neurons which we think are a part of the glucoreceptor. We have studied the system by making antiglucoreceptor antibodies. When we applied these antibodies to the VMH neurons, their electrical activity decreased.

Van Houten: But that still doesn't rule out the possibility that the glucose might be acting intracellularly through some metabolic mechanism.

Oomura: I don't think that is the case, for when we apply phlorizin, it has the same effect as glucose. This suggests that glucose binding is enough for excitation. More recently, we have been able to get intracellular recordings. These neurons are very small, less than 10 microns. How- ever, using a new technique, we have finally succeeded. When we applied glucose, there was an instant depolarization.

Van Houten: But couldn't the glucose be acting by influencing a second neuron and secondarily activating the neuron from which you're recording via a neurotransmitter?

Oomura: Yes, that's still possible, although I doubt it. To be certain, we should do something like increase the magnesium concentration to prevent transmitter release.

Woods: What effect does alloxan have on your glucoreceptive neurons?

Oomura: We have not yet tried alloxan.

B. Jeanrenaud: What is the nature of the glucoreceptor protein? Have you characterized it at all? 
Oomura: All we know is that there must be some amino acid sequence since we can develop antibodies to it.

Porte: How do you get these antibodies?

Oomura: By injecting homogenized neural material from the glucose sensitive area of the VMH into rabbits. We also made antibodies to rat cortex and caudate as a control, and these had no effect on the VMH neurons. The details of the procedure have been published in the Proceedings of the Second International Obesity Congress, edited by Dr. Bray.

Nicolaidis: How do you purify your antibody preparation?

Oomura: After we have obtained the antisera to the different areas of the brain, we take out the common factors by precipitation. We finally use the fraction which precipitates only with VHM homogenate.

Fernstrom: If I've got this correct, you hypothesize that when the animal has plenty of glucose, it effects these special neurons you have studied; and when the animal has inadequate supplies of glucose but other substrates like betahydroxybutyrate are available, the same neurons are stimulated in the opposite direction and this tells the animal that it ought to eat. That's a very interesting hypothesis.
Have you ever applied your glucose or other compounds systemically and measured the effect on these neurons? This would seem much more physiological.

Oomura: Actually, in our first experiments, back in 1964, we applied glucose or insulin into the carotid arteries, and we were able to measure the same changes in these neurons. But these effects might have been due to activation of other neurons than those from which we were recording so we have gone to studying more direct effects.

Fernstrom: Your animals are always anesthetized. It would be interesting to try starved animals where glucose is low and betahydroxybutyrate is high, and to look at lactation where betahydroxybutyrate is high but glucose is not necessarily low. Have you tried these?

Oomura: We have not yet tried the experiments you mention. We have done some studies on the awake, leverpressing monkey. We have applied glucose and other substances directly into the brain and gotten comparable results. So we know that the anesthesia is not having an effect.

Seydoux: What are the proportions of various neural types in the LH area?

Oomura: Around 20 or $25 \%$ have glucoreceptors. Another $20 \%$ are osmosensitive neurons. 